\title{
A New Passive/Active Hybrid Headset for a Helicopter Application
}

\author{
M. Winberg, S. Johansson, T. Lagö, I. Claesson \\ University of Karlskrona/Ronneby, Department of Signal Processing, S-372 25 Ronneby, Sweden
}

In helicopters, the low frequency noise generated by the rotors and engines often masks and jeopardizes safe communication. In addition, pilots are likely to suffer from damage to their hearing due to the high sound levels in the headset produced to overcome the noise caused by increased speaker levels. A feasible approach is to reduce the low frequency noise by using active techniques combined with a method for reducing the noise in the intercom microphone signal, with lower speaker levels as a result. Helicopter noise consists of tonal components embedded in broadband noise. In order to achieve an efficient attenuation of the primary noise inside the headset, a combination of a digital feedforward controller and an analog feedback controller is employed. Spectral Subtraction is used to suppress the background noise in speech signals. This paper evaluates a combination of the two techniques and their application to real data.

\section{INTRODUCTION}

There are substantial noise levels in the Super Puma helicopter, especially at low frequency. ${ }^{1}$ The noise level is not normally harmful to hearing, although low frequency noise generated by the engines and rotors (main and tail rotor) masks and corrupts speech. Noise with a dominant frequency distribution just below, or within the lower frequency range for speech, degrades speech recognition and intelligibility. ${ }^{2}$ For helicopter pilots it is important to hear radio communication correctly during flight. Pilots thus tend to select the maximum hearing volume in the communication system. The sound levels produced are harmful to the ear, inducing fatigue and loss of hearing. This phenomenon is denoted Noise Induced Hearing Loss (NIHL), a condition in which high sound pressure levels (SPLs) are possible. The level of risk NIHL that occurs depends on the frequency content of the noise.

In order to increase speech intelligibility, the noise level inside the earcups must be reduced. Since the noise has low frequency characteristics, it cannot be substantially reduced by employing passive techniques since the passive earcups cannot be made large enough, due to the fact that they must fit inside the helmet. The helicopter pilots and the rescue personnel are required to wear a helmet for safety reasons. Had there been no such stipulation, larger earcups such as the Bilsom 747 which provide good low frequency attenuation could be used. A more feasible approach is to reduce the noise by employing Active Noise Control (ANC). As Figure 1 shows, noise up to $100 \mathrm{~Hz}$ inside the helicopter, is normally dominated by tonal components while the noise is more broadband between $100 \mathrm{~Hz}$ to $400 \mathrm{~Hz}$. The total sound pressure level inside the helicopter is $103 \mathrm{~dB}(\mathrm{~A})$.

The approach in this paper uses a hybrid ANC headset which combines both feedforward and feedback ANC techniques. ${ }^{3}$ The adaptive feedforward controller is based on a digital system, while the feedback system is based on an analog system. The principle of the hybrid headset is depicted in Fig. 2. This type of ANC headset is used in order to improve noise attenuation. The feedback controller reduces broadband noise, while the feedforward controller reduces tonal components (harmonics of the main and tail rotor).

The feedback controller is based on a commercial analog headset. Pure analog feedback technique will not be discussed in the paper. Here, the approach focuses on the adaptive algorithm used in the digital feedforward controller, as well as the combined performance of the hybrid headset.

Even though the hybrid headset efficiently reduces the low frequency noise inside the earcups, high noise levels are still produced in the earcups through the intercom microphones used for communication. It is thus desirable that the noise in the speech signal also be reduced. Speech enhancement and speech recognition are either model-based, where typical solutions include Markov Models, Predictors and Lattice filters; ${ }^{4}$ or non-parametric, based on a spectral description of speech such as the Short-Term Spectrum or Cepstral techniques. ${ }^{5}$ Spectral Subtraction (SS) is a suitable method which uses a single microphone. This non-parameter method is based on the additive properties of the magnitudes of the FFT coefficients of the noise. SS has been combined with the hybrid headset as depicted in Fig. 2.

\section{HELICOPTER NOISE}

In order to analyze the frequency components of helicopter noise, several noise measurements were performed during flight. The noise was recorded inside the helicopter using a microphone situated between the passenger seats, in the vicinity of the right rear door (see Fig. 3). When hovering, this door is open.

The Super Puma helicopter has one main rotor and one tail rotor, as well as several gear boxes. There are four blades on the main rotor and five on the tail rotor. Figure 4 shows a typical noise power spectrum inside the cabin during flight 